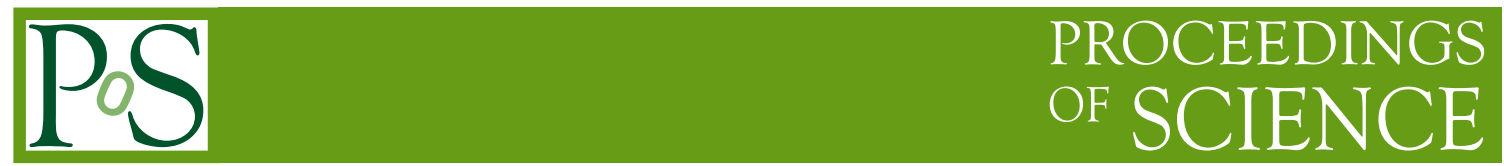

\title{
Top and EW Physics at the LHeC
}

\section{Zhiqing Zhang*i}

Laboratoire de l'Accélérateur Linéaire, Univ. Paris-Sud 11 et IN2P3/CNRS, France

E-mail: zhang@lal.in2p3.fr

The LHeC is a proposed upgrade of the LHC to study ep/eA collisions in the TeV regime, by adding a $60 \mathrm{GeV}$ electron beam through an energy recovery linac. In $e p$, high precision top and electroweak physics can be performed, such as measurements of anomalous top couplings, light quark couplings to the $Z$ boson and the energy dependence of the weak mixing angle $\sin ^{2} \theta_{W}$, for which simulation studies are presented.

The European Physical Society Conference on High Energy Physics

22-29 July 2015

Vienna, Austria

* Speaker.

${ }^{\dagger}$ for the LHeC Study Group 


\section{Introduction}

Deep inelastic scattering (DIS) of a point-like lepton beam over a hadron has played a central role in establishing the quark-parton model and QCD starting with fixed target experiments in the late 1960s at SLAC. Later the Gargamelle neutrino-nucleon experiment at CERN has discovered weak neutral currents. HERA, operated at DESY from 1992 to 2007, was the only ep collider of the world. It has extended the study of the proton structure and quark-gluon interaction dynamics up to a centre-of-mass energy $(\sqrt{s})$ of $320 \mathrm{GeV}$ corresponding to an extension by two orders of magnitude towards both higher negative four-momentum transfer squared $Q^{2}$ and lower Bjorken $x$ in comparison with the kinematic region covered by the fixed target experiments.

The $\mathrm{LHeC}$, if realised by adding to the LHC a separate $9 \mathrm{~km}$ racetrack-shaped recirculating superconducting energy recovery linac providing a polarised electron (possibly also positron) beam of $60 \mathrm{GeV}$, will be a new ep collider of $1.3 \mathrm{TeV}$, running in parallel with the high luminosity phase of the LHC. It has a rich and complementary physics programme to the LHC [1,2]. It would enable new precision studies of QCD in general and the precision determination of parton distributions functions (PDFs) in a largely extended kinematic region in particular. It has the potential to reveal new QCD dynamics in an unexplored low $x$ regime where the DGLAP evolution equations may no longer be valid as the latest QCD analysis of the newly combined inclusive neutral and charged current (NC and $\mathrm{CC}$ ) cross sections at HERA may indicate [3]. It would also provide additional and sometimes unique ways for studying top and electroweak (EW) physics as well as Higgs and physics beyond the Standard Model (BSM).

This talk focuses on some of the selected topics on top and EW physics at the $\mathrm{LHeC}$ and the writeup is organised as follows. In Sec. 2, expected limits on anomalous Wtb couplings from the single top production are presented as an exemple. In Sec. 3, the expected precision determination of light quark couplings to the $Z$ boson and the scale dependence of the weak mixing angle $\sin ^{2} \theta_{W}$ based either on the inclusive NC cross section measurements or on polarisation asymmetries of the NC interactions are shown, followed by a summary in Sec. 4.

\section{Top physics}

The top quark is the heaviest particle in the SM, which is believed to be most sensitive to BSM physics. It has not been studied so far by any DIS experiments because of the kinematic limit or too small cross section. Therefore the $\mathrm{LHeC}$ will be the first DIS experiment capable to study the directly produced single top quark and top pairs in $\mathrm{CC}$ and $\mathrm{NC}$ interactions, respectively.

In the five flavour scheme, the single top-quark production cross section of the $2 \rightarrow 2 t$-channel process $e^{-} p \rightarrow \bar{t} v_{e}+X$ with $\bar{t} \rightarrow W^{-} b$ at $\sqrt{s}=1.3 \mathrm{TeV}$ is predicted to be around $2 \mathrm{pb}$ for un polarised electron beam and increases by a factor of $1+P_{e}$ with $P_{e}$ being the degree of the longitudinal polarisation of the beam [4]. This cross section value is comparable with that of the Tevatron and smaller by about two orders of magnitude than the LHC at $14 \mathrm{TeV}$ [5]. The LHeC has however a much cleaner environment due to the absence of pile-up and underlying events. Therefore this process can be used for many precision measurements within the SM, such as the bottom-quark distribution of the proton, the CKM matrix element $V_{t b}$, the $t$-quark polarisation and the $W$ boson helicity. It can also be used to study deviations from the SM such as the anomalous couplings $W t b$. 
In addition, the single top production in the NC protoproduction can be used to study top quark flavour changing neutral current couplings $t q \gamma$ with $q$ being a light quark [1].

The top pair events are also produced at the $\mathrm{LHeC}$ in $\mathrm{NC}$ interactions. Even though the rate is lower than at the LHC, the potential for a better measurement of $t$ t $\gamma$ than LHC is good [6] as in the $t \bar{t}$ photoproduction at the $\mathrm{LHeC}$, the highly energetic incoming photon couples only to the $t$ quark so that the cross section depends directly on the $t t \gamma$ vertex, whereas at the LHC the vertex is probed through $t \bar{t} \gamma$ production, where the outing going photon could come from other charged sources such as the top decays products. The DIS regime of $t \bar{t}$ production will also be able to probe the $t t Z$ coupling though with less sensitivity.

A detailed study was performed in [4] to evaluate the expected accuracy of measuring the anomalous $W t b$ couplings at the $\mathrm{LHeC}$ based on the single anti-top quark production in $e^{-} p$ collisions in a model independent way by means of the following effective CP conserving Lagrangian [4]

$$
\mathscr{L}_{W t b}=\frac{g}{\sqrt{2}}\left[W_{\mu} \bar{t} \gamma^{\mu}\left(V_{t b} f_{1}^{L} P_{L}+f_{1}^{R} P_{R}\right) b-\frac{1}{2 m_{W}} W_{\mu v} \bar{t} \sigma^{\mu v}\left(f_{2}^{L} P_{L}+f_{2}^{R} P_{R}\right) b\right]+\text { h.c. }
$$

where $f_{1}^{L}\left(\equiv 1+\Delta f_{1}^{L}\right)$ and $f_{1}^{R}$ are left- and right-handed vector couplings, $f_{2}^{L, R}$ are left- and righthanded tansor couplings, $W_{\mu v}=\partial_{\mu} W_{v}-\partial_{v} W_{\mu}, P_{L, R}=\frac{1}{2}\left(1 \mp \gamma_{5}\right)$ are left- and right-handed projection operators, $\sigma^{\mu v}=i / 2\left(\gamma^{\mu} \gamma^{v}-\gamma^{v} \gamma^{\mu}\right)$ and $g=2 / \sin \theta_{W}$. In the SM, $f_{1}^{L} \equiv 1$ and $\Delta f_{1}^{L}=f_{1}^{R}=$ $f_{2}^{L, R} \equiv 0$.

Several analyses were performed using a simulated event sample corresponding to an integrated luminosity of $100 \mathrm{fb}^{-1}$ for three different systematic uncertainties of $1 \%, 5 \%$ and $10 \%$. One of them was based on a $\chi^{2}$ analysis using differential distributions of a few relevant kinematic variables in the leptonic and hadronic decay modes, respectively. Contours at $68 \%$ and $95 \%$ confidence level (CL) on two dimensional plane for any coupling combination were presented. One example is shown in Fig. 1. The corresponding results in comparison with other results from Tevatron, LHC and indirect one from $B$ decays are shown in Table 1. The conservative LHeC limits are thus competitive with or better than similar results from other determinations.
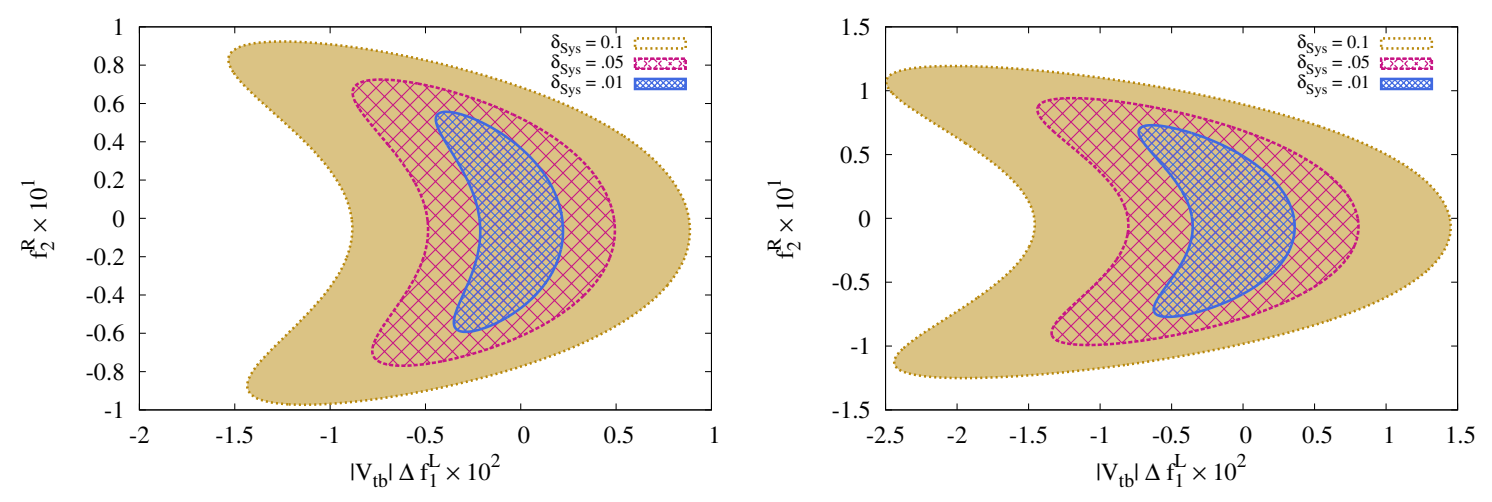

Figure 1: Contours at (left) $68 \%$ and (right) $95 \% \mathrm{CL}$ on the plane of $\left|V_{t b}\right| \Delta_{1}^{L}$ and $f_{2}^{R}$ for a systematic error of $1 \%, 5 \%$ and $10 \%$ on a sample with an integrated luminosity of $100 \mathrm{fb}^{-1}$ (figures taken from Ref. [4]). 


\begin{tabular}{l|cccc} 
Upper limit (95\% CL) & $\left|\Delta f_{1}^{L}\right|$ & $\left|f_{1}^{R}\right|$ & $\left|f_{2}^{L}\right|$ & $\left|f_{2}^{R}\right|$ \\
\hline LHeC [4] & $0.005-0.03$ & $0.01-0.1$ & $0.01-0.1$ & $0.01-0.1$ \\
D0 [7] & & 0.548 & 0.324 & 0.347 \\
LHC [8] & $0.03-0.06$ & $0.22-0.34$ & $0.06-008$ & $0.06-0.08$ \\
$B$ decays [9] & {$[-0.13,0.03]$} & {$[-0.0007,0.0025]$} & {$[-0.0013,0.0004]$} & {$[-0.15,0.57]$} \\
\hline
\end{tabular}

Table 1: Comparison of expected upper limits at $95 \% \mathrm{CL}$ at the $\mathrm{LHeC}\left(100 \mathrm{fb}^{-1}\right.$, hadronic modes, $\delta_{\mathrm{sy}}=$ $0.01-0.1)$ [4] with the actual limits from D0 (5.4 $\mathrm{fb}^{-1}, W$-helicity, single top) [7] and expected limits at the LHC (100 $\left.\mathrm{fb}^{-1}, \gamma p \rightarrow W t X\right)[8]$ and $B$ decays (indirect) [9].

\section{EW physics}

Inclusive NC and CC DIS interactions are two main processes which can be measured at the LHeC with high precision providing primary source not only for precision QCD studies but also for EW physics. Three examples are briefly presented in this section.

The first example concerns a precision measurement of vector and axial-vector weak NC couplings of the $Z$ boson to light quarks $v_{q}$ and $a_{q}$. They were determined together with PDFs in a combined EW and QCD analysis of simulated inclusive NC and CC cross section data samples following Ref. [10]. This is possible since the double differential NC cross section $\frac{\mathrm{d}^{2} \sigma_{\mathrm{NC}}}{\mathrm{d} x Q^{2}}$ in e.g. $e^{-} p$ collisions may be expressed in terms of three structure functions as $\frac{2 \pi \alpha^{2}}{x Q^{2}}\left[Y_{+} \tilde{F}_{2}+Y_{-} \tilde{F}_{3}-y^{2} \tilde{F}_{L}\right]$, where $\alpha$ is the electromagnetic fine structure constant and $Y_{ \pm}=1 \pm(1-y)^{2}$ with $y=Q^{2} /(x s)$ being the electron inelasticity. The generalised structure $\tilde{F}_{2}$ can be decomposed as $F_{2}+P_{e} a_{e} \kappa_{Z} F_{2}^{\gamma Z}+a_{e}^{2} \kappa_{Z}^{2} F_{2}^{Z}$ corresponding to $\gamma$ exchange, $\gamma Z$ interference and $Z$ exchange contributions. In this expression, $\kappa_{Z}^{-1}=\frac{2 \sqrt{2} \pi \alpha}{G_{F} M_{Z}^{2}} \frac{Q^{2}+M_{Z}^{2}}{Q^{2}}$ and $a_{e}$ is the axial-vector coupling of the electron (due to the smallness of the vector coupling $v_{e}$, terms proportional to $v_{e}$ have been omitted). Similarly $x \tilde{F}_{3}=-a_{e} \kappa_{Z} x F_{3}^{\gamma Z}-$ $P_{e} a_{e}^{2} \kappa_{Z}^{2} x F_{3}^{\gamma Z}$. These different structure functions can be further expressed in terms of PDFs $q, \bar{q}$ and the light quark couplings $v_{q}$ and $a_{q}$ as $\left[F_{2}, F_{2}^{\gamma Z}, F_{2}^{Z}\right]=x \sum_{q}\left[e_{q}^{2}, 2 e_{q} v_{q}, v_{q}^{2}+a_{q}^{2}\right]\{q+\bar{q}\}$ and $\left[x F_{3}^{\gamma Z}, x F_{3}^{Z}\right]=2 x \sum_{q}\left[e_{q} a_{q}, v_{a} a_{q}\right]\{q-\bar{q}\}$, where $e_{q}$ being the electronic charge of quark $q$. The longitudinal structure function $\tilde{F}_{L}$ does not contribute at LO. The CC cross section is independent of these couplings but its inclusion in the fit helps to constrain the PDFs.

In Ref. [1], different scenarios were considered. The results of one of these, corresponding to $e^{ \pm}$beams of $50 \mathrm{GeV}$ with a longitudinal polarisation of $40 \%$ colliding with a proton beam of $7 \mathrm{TeV}$ for an integrated luminosity of $1 \mathrm{fb}^{-1}$ per beam, are shown in Fig. 2 in comparison with similar determinations from other experiments. The expected precision at the $\mathrm{LHeC}$ is indeed much better and any significant deviation from the SM expectations can thus be observed with this analysis.

The second example is on the scale dependence of the weak mixing angle $\sin ^{2} \theta_{W}$ obtained from a projected measurement of the polarisation asymmetry $A^{-}=\frac{\sigma_{\mathrm{NC}}^{-}\left(P_{R}\right)-\sigma_{\mathrm{NC}}^{-}\left(P_{L}\right)}{\sigma_{\mathrm{NC}}^{-}\left(P_{R}\right)+\sigma_{\mathrm{NC}}^{-}\left(P_{L}\right)} \simeq \frac{\kappa_{Z} a_{e}\left(P_{L}-P_{R}\right)}{2} \frac{F_{2}^{\gamma Z}}{F_{2}}$ assuming a left-handed $\left(P_{L}\right)$ or right-handed $\left(P_{R}\right)$ polarisation of $80 \%$ and an integrated luminosity of $10 \mathrm{fb}^{-1}$ per polarisation state [1]. The results are compared in Fig. 3 (left) with other determinations at different energies. The $\mathrm{LHeC}$ measurements are precise and cover a large energy range. It should be mentioned that the $\mathrm{NC}$ and $\mathrm{CC}$ cross section ratio is also sensitive to $\sin ^{2} \theta_{W}$, provided that the PDFs related uncertainty is under control [1]. 

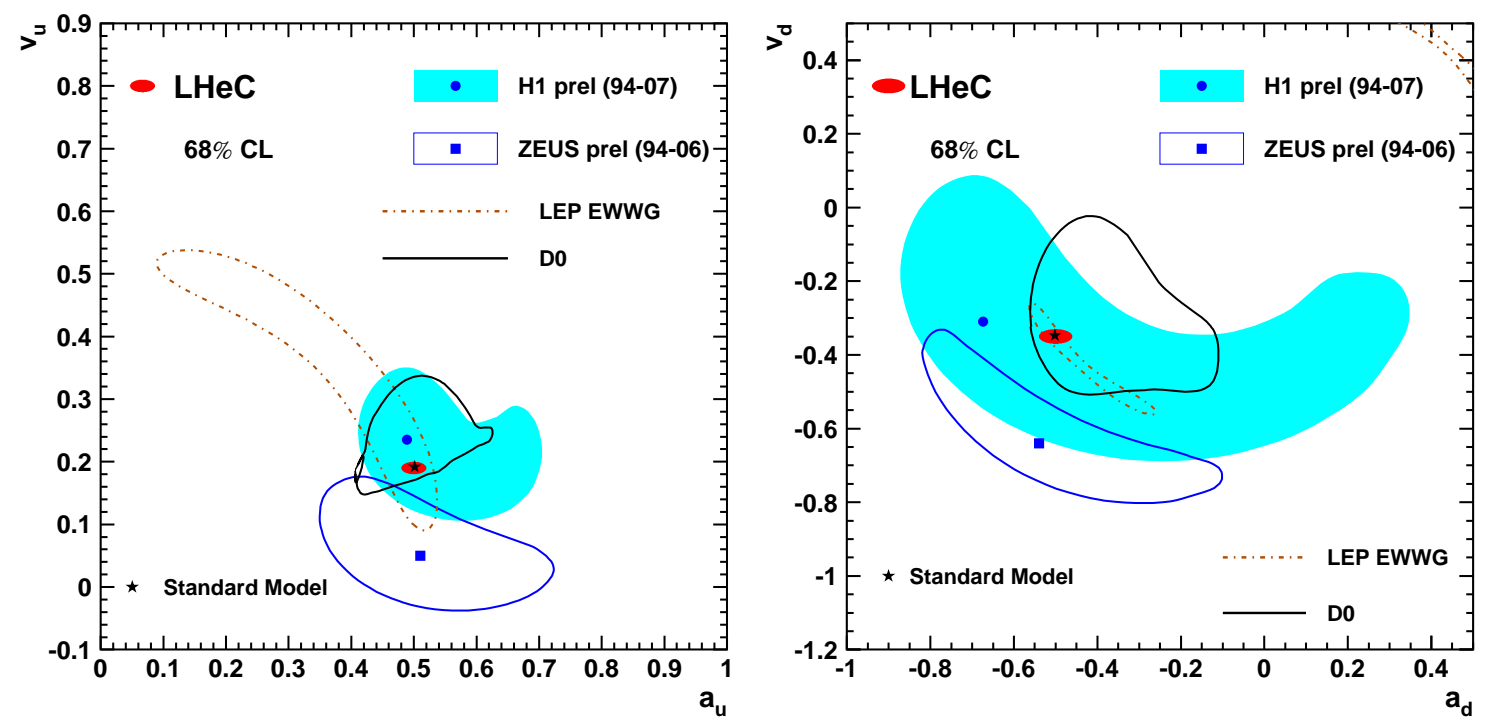

Figure 2: Determination of the vector and axial-vector weak neutral current couplings of the light quarks by LEP [11], D0 [12], H1 [13] and ZEUS [14], compared with the simulated prospects for the LHeC [1].
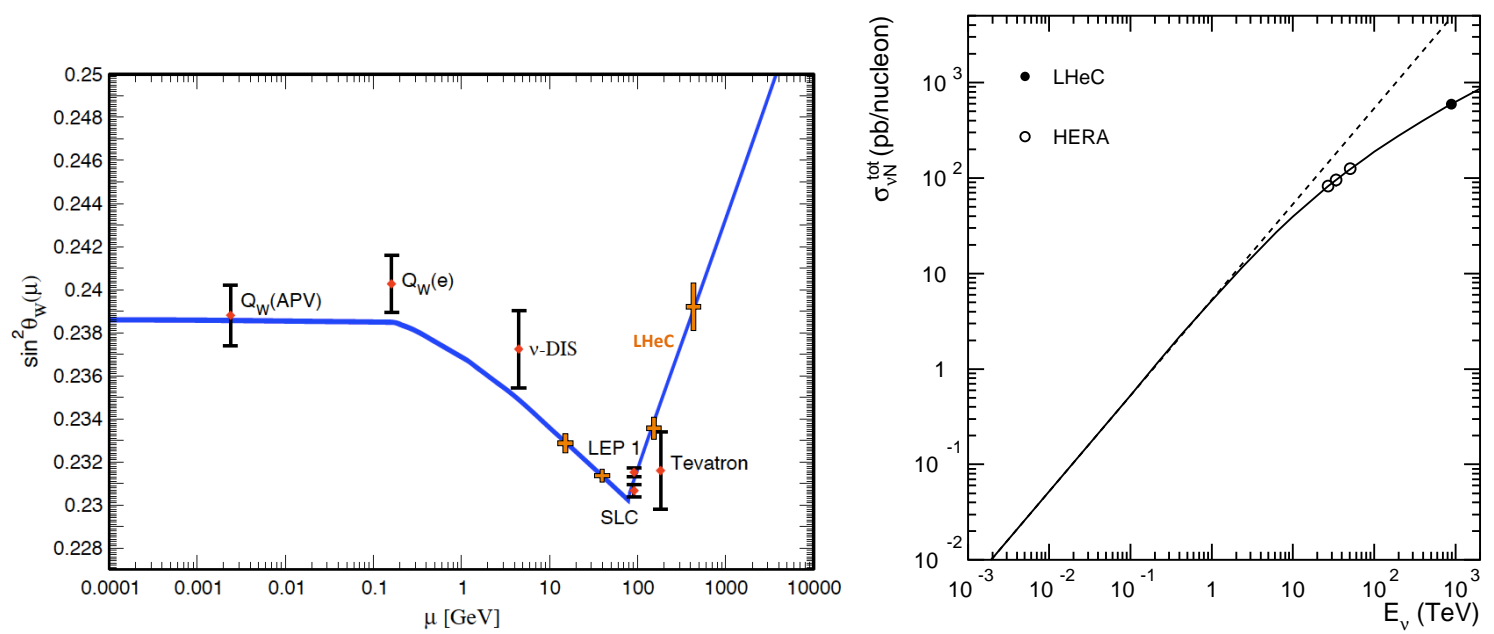

Figure 3: Left: dependence of the weak mixing angle on the energy scale $\mu$ from [1]. Right: energy dependence of the $v N$ cross section. The open points up to $50 \mathrm{TeV}$ correspond to the expected precision of the HERA measurements and the solid point corresponds to an expected measurement at the LHeC. The full line represents the predicted cross section including the $W$ propagator while the dashed line is a linear extrapolation from low energy measurements.

The third example is on a measurement of the CC total cross section (Fig. 3 (right)). The LHeC measurement together with those from HERA illustrates in a spectacular way the impact of the propagator mass of the $W$ boson on the CC cross section. The dependence on the polarisation of the $\mathrm{CC}$ total cross section can further be used to set a stringent lower mass limit on a right-handed $W$ boson following Ref. [15]. 


\section{Summary}

A few selected examples of the $\mathrm{LHeC}$ measurements from top and $\mathrm{EW}$ sectors clearly demonstrate that the realisation of the $\mathrm{LHeC}$ can greatly enhance the physics programme and discovery potential of the LHC in a complementary manner. Indeed the LHeC is also considered as the next machine for studying the Higgs boson and a luminosity upgrade by a factor of 10 reaching $10^{34} \mathrm{~cm}^{-2} s^{-1}$ is under study [16], compared to the Conceptional Design Report [1]. More studies are desirable from both theoretical and experimental communities, which will certainly reveal even larger potential of the $\mathrm{LHeC}$.

\section{References}

[1] J. L. Abelleira Fernandez et al. [LHeC Study Group], "A Large Hadron Electron Collider at CERN", J. Phys. G. 39 (2012) 075001, arXiv:1206.2913 [physics.acc-ph].

[2] J. L. Abelleira Fernandez et al. [LHeC Study Group Collaboration], "On the Relation of the LHeC and the LHC", arXiv:1211.5102 [hep-ex].

[3] F. D. Aaron et al. [H1 and ZEUS Collaborations], JHEP 1001 (2010) 109, arXiv:0911.0884 [hep-ex].

[4] S. Dutta, A. Goyal, M. Kumar and B. Mellado, arXiv:1307.1688 [hep-ph].

[5] N. Kidonakis, arXiv:1509.02528 [hep-ph].

[6] A. O. Bouzas and F. Larios, Phys. Rev. D 88 (2013) 9, 094007 [arXiv:1308.5634 [hep-ph]].

[7] V. M. Abazov et al. [D0 Collaboration], Phys. Lett. B 713 (2012) 165 [arXiv:1204.2332 [hep-ex]].

[8] B. Sahin and A. A. Billur, Phys. Rev. D 86 (2012) 074026 [arXiv:1210.3235 [hep-ph]].

[9] B. Grzadkowski and M. Misiak, Phys. Rev. D 78 (2008) 077501 [Phys. Rev. D 84 (2011) 059903] [arXiv:0802.1413 [hep-ph]].

[10] A. Aktas et al. [H1 Collaboration], Phys. Lett. B 632 (2006) 35 [hep-ex/0507080].

[11] S. Schael et al. [ALEPH and DELPHI and L3 and OPAL and SLD and LEP Electroweak Working Group and SLD Electroweak Group and SLD Heavy Flavour Group Collaborations], Phys. Rept. 427 (2006) 257 [hep-ex/0509008].

[12] V. M. Abazov et al. [D0 Collaboration], Phys. Rev. D 84 (2011) 012007 [arXiv:1104.4590 [hep-ex]].

[13] Z. Zhang (for the H1 Collaboration), Combined electroweak and QCD fits including NC and CC data with polarised electron beam at HERA-2, PoS DIS2010 (2010) 056.

[14] ZEUS Collaboration, ZEUS-prel-07-027.

[15] A. Aktas et al. [H1 Collaboration], Phys. Lett. B 634 (2006) 173 [hep-ex/0512060].

[16] O. Bruening and M. Klein, Mod. Phys. Lett. A 28 (2013) 16, 1330011 [arXiv:1305.2090 [physics.acc-ph]]. 